\title{
Spin-Density Correlations and Short-Range Order in Metals above the Curie Temperature
}

\author{
Nikolai B. Melnikov ${ }^{1, \star}$, Boris I. Reser ${ }^{2, \star \star}$, and Georgii V. Paradezhenko ${ }^{1, \star \star \star}$ \\ ${ }^{1}$ Lomonosov Moscow State University, Moscow, Russia \\ ${ }^{2}$ Mikheev Institute of Metal Physics, RAS, Ekaterinburg, Russia
}

\begin{abstract}
In the dynamic spin-fluctuation theory, we calculate the effective and local magnetic moments and spatial spin-density correlator. Our theoretical results are demonstrated by the example of bcc Fe. The effective and local moments are found in good agrement with results of polarized neutron scattering experiment over a wide temperature range. The calculated short-range order is small (up to $5 \AA$ ) and slowly decreases with temperature.
\end{abstract}

\section{Introduction}

Polarized neutron scattering experiments $[1,2]$ point to the existence of large short-range order (SRO) of about $20 \AA$ in metals above the Curie temperature, but the interpretation of the experiment based on the spin waves is controversial.

Static spin-fluctuation theories all support the existence of SRO, but there is no agreement about the spin correlation length (see, e.g., [3]). The dynamic theories based on the local approximation $[4,5]$ do not allow to estimate the SRO quantitatively.

The dynamic spin-fluctuation theory (DSFT) [6-8] takes into account both quantum nature and nonlocal character of the spin fluctuations. For selected temperatures, calculations of the spin-density correlator and effective moment in the dynamic approximations of spinfluctuation theory were carried out by $[6,9]$.

We use the DSFT to calculate the spin correlator as a function of distance and temperature; calculate the effective moment as a function of wavevector and temperature and compare the results with experiment [10-12]. Our theoretical results are demonstrated in the example of bcc Fe.

\section{Spin correlations in metals}

From a theoretical point of view, we are interested in the spatial correlator $\left\langle s^{\alpha}(\mathbf{r}) s^{\alpha}(0)\right\rangle$ of the spin density

$$
s^{\alpha}(\mathbf{r})=\sum_{i=1}^{N_{\mathrm{e}}} s_{i}^{\alpha} \delta\left(\mathbf{r}-\mathbf{r}_{i}\right), \quad \alpha=x, y, z,
$$

where $\mathbf{s}_{i}$ is the spin of the $i$ th electron and $N_{\mathrm{e}}$ is the number of electrons. In the experiment, the measured scattering is

\footnotetext{
^e-mail: melnikov@cs.msu.ru

$\star \star$ e-mail: reser@imp.uran.ru

$\star \star \star$ e-mail: gparadezhenko@cs.msu.ru
}

expressed in terms of the square of the effective moment

$$
M^{2}(q)=\frac{1}{N}\left\langle\mathbf{s}_{\mathbf{q}} \mathbf{s}_{-\mathbf{q}}\right\rangle g^{2} \mu_{\mathrm{B}}^{2},
$$

where $N$ is the number of atoms and

$$
s_{\mathbf{q}}^{\alpha}=\int s^{\alpha}(\mathbf{r}) \mathrm{e}^{-\mathrm{iqr}} \mathrm{d} \mathbf{r}
$$

is the Fourier transform of the spin-density $s^{\alpha}(\mathbf{r})$.

From the spin correlator we can obtain various magnetic characteristics. For instance, integration over the Wigner-Seitz cell of the volume $\Omega_{\mathrm{WS}}$ gives the square of the local spin moment

$$
s_{\mathrm{L}}^{2}=\iint_{\mathrm{WS}}\left\langle\mathbf{s}(\mathbf{r}) \mathbf{S}\left(\mathbf{r}^{\prime}\right)\right\rangle \mathrm{d} \mathbf{r} \mathrm{d} \mathbf{r}^{\prime} \approx \Omega_{\mathrm{WS}}^{2}\langle\mathbf{s}(0) \mathbf{S}(0)\rangle .
$$

The correlator can be calculated by inverting the Fourier transformation (2):

$$
\left\langle s^{\alpha}(\mathbf{r}) s^{\alpha}(0)\right\rangle=\frac{1}{V} \sum_{\mathbf{q}}\left\langle s_{\mathbf{q}}^{\alpha} s_{-\mathbf{q}}^{\alpha}\right\rangle \mathrm{e}^{\mathrm{i} \mathbf{q r}},
$$

where $V=N \Omega_{\mathrm{WS}}$ is the volume of the crystal. Replacing the sum over the Brillouin zone by the integral over a sphere of equal volume with the radius $q_{\mathrm{B}}$, we obtain [12]

$$
\left\langle s^{\alpha}(\mathbf{r}) s^{\alpha}(0)\right\rangle=\frac{1}{2 \pi^{2} N \Omega_{\mathrm{WS}}} \int_{0}^{q_{\mathrm{B}}}\left\langle s_{\mathbf{q}}^{\alpha} s_{-\mathbf{q}}^{\alpha}\right\rangle \frac{q \sin (q r)}{r} \mathrm{~d} q .
$$

Passing to the limit $r \rightarrow 0$ in expression (4) and substituting the result in (3), we come to

$$
s_{\mathrm{L}}^{2}=\frac{\Omega_{\mathrm{WS}}}{2 \pi^{2} N} \int_{0}^{q_{\mathrm{B}}}\left\langle\mathbf{s}_{\mathbf{q}} \mathbf{s}_{-\mathbf{q}}\right\rangle q^{2} \mathrm{~d} q .
$$

Using formula (1), we can write the square of the local magnetic moment $m_{\mathrm{L}}=s_{\mathrm{L}} g \mu_{\mathrm{B}}$ as

$$
m_{\mathrm{L}}^{2}=\frac{4 \pi}{\Omega_{\mathrm{BZ}}} \int_{0}^{q_{\mathrm{B}}} q^{2} M^{2}(q) \mathrm{d} q
$$


where $\Omega_{\mathrm{BZ}}=(2 \pi)^{3} / \Omega_{\mathrm{WS}}$ is the volume of the Brillouin zone.

Calculation of $M^{2}(q)$ by formula (1) requires the spin correlator $\left\langle s_{\mathbf{q}}^{\alpha} s_{-\mathbf{q}}^{\alpha}\right\rangle$. We use the fluctuation-dissipation theorem:

$$
\left\langle s_{\mathbf{q}}^{\alpha} s_{-\mathbf{q}}^{\alpha}\right\rangle=\frac{1}{2 \pi} \int B(\varepsilon) \operatorname{Im} \chi_{\mathbf{q}}^{\alpha}(\varepsilon) \mathrm{d} \varepsilon
$$

where

$$
\chi_{\mathbf{q}}^{\alpha}(\omega)=\frac{2 \mathrm{i}}{\hbar} \int_{0}^{\infty}\left\langle\left[s_{\mathbf{q}}^{\alpha}(t), s_{-\mathbf{q}}^{\alpha}\right]\right\rangle \mathrm{e}^{\mathrm{i} \omega t} \mathrm{~d} t
$$

is the dynamic susceptibility (in units of $\frac{1}{2} g^{2} \mu_{\mathrm{B}}^{2}$ ) and $B(\varepsilon)=\left(\mathrm{e}^{\varepsilon / T}-1\right)^{-1}$ is the Bose function (for details, see, e.g., [13]).

In the DSFT, the enhanced susceptibility is given by

$$
\chi_{\mathbf{q}}^{\alpha}(\varepsilon)=\frac{\chi_{\mathbf{q}}^{0 \alpha}(\varepsilon)}{1-\tilde{u} \chi_{\mathbf{q}}^{0 \alpha}(\varepsilon)}
$$

where $\chi_{\mathbf{q}}^{0 \alpha}(\varepsilon)$ is the unenhnaced susceptibility of the noninteracting electrons in the fluctuating field and $\tilde{u}=u / N$ is the Fourier transform of the effective interaction constant $u$. Since the Bose function $B(\varepsilon)$ is localized around $\varepsilon=0$, the unenhnaced susceptibility $\chi_{\mathbf{q}}^{0 \alpha}(\varepsilon)$ is replaced by its Taylor series in $\varepsilon$. Then

$$
\left\langle s_{\mathbf{q}}^{\alpha} s_{-\mathbf{q}}^{\alpha}\right\rangle=\frac{T}{2 \tilde{u} \lambda_{\mathbf{q}}^{\alpha}} \frac{2}{\pi} \arctan \frac{\tilde{u} \varphi_{\mathbf{q}}^{\alpha} \pi^{2} T}{6 \lambda_{\mathbf{q}}^{\alpha}}
$$

where $\lambda_{\mathbf{q}}^{\alpha}=1-\tilde{u} \chi_{\mathbf{q}}^{0 \alpha}(0)$ and $\varphi_{\mathbf{q}}^{\alpha}=\mathrm{d} \chi_{\mathbf{q}}^{0 \alpha}(0) / \mathrm{d} \varepsilon$. To take the mode-mode interaction into account, the static susceptibility $\chi_{\mathbf{q}}^{0 \alpha}(0)$ is interpolated between the homogeneous $\chi_{0}^{0 \alpha}(0)$ and local $\chi_{\mathrm{L}}^{0 \alpha}(0)$ susceptibilities. Thus,

$$
\lambda_{\mathbf{q}}^{\alpha}=\lambda_{0}^{\alpha}+\left(\lambda_{\mathrm{L}}^{\alpha}-\lambda_{0}^{\alpha}\right) q^{2} / \overline{q^{2}}
$$

where $\lambda_{\mathrm{L}}^{\alpha}=1-u \chi_{\mathrm{L}}^{0 \alpha}(0)$ and $\overline{q^{2}}$ is the average over the Brillouin zone [7]. For simplicity, $\varphi_{\mathbf{q}}^{\alpha}$ is replaced by its mean value. Then the spin correlator is given by [12]

$$
\left\langle s_{\mathbf{q}}^{\alpha} s_{-\mathbf{q}}^{\alpha}\right\rangle=\frac{N T}{2 u \lambda_{\mathrm{L}}^{\alpha}} \frac{1}{a_{\alpha}^{2}+b_{\alpha}^{2}\left(q / q_{\mathrm{B}}\right)^{2}} \frac{2}{\pi} \arctan \frac{c_{\alpha}}{a_{\alpha}^{2}+b_{\alpha}^{2}\left(q / q_{\mathrm{B}}\right)^{2}},
$$

where the coefficients $a_{\alpha}, b_{\alpha}, c_{\alpha}$ and $\lambda_{\mathrm{L}}^{\alpha}$ are calculated selfconsistently at each $T$.

Now we consider high-temperatures $\left(T \gg T_{\mathrm{C}}\right)$. Approximating the Bose function $B(\varepsilon) \approx T / \varepsilon$, we write the fluctuation-dissipation theorem (7) as

$$
\left\langle s_{\mathbf{q}}^{\alpha} s_{-\mathbf{q}}^{\alpha}\right\rangle=\frac{T}{2 \pi} \int \frac{\operatorname{Im} \chi_{\mathbf{q}}^{\alpha}(\varepsilon)}{\varepsilon} \mathrm{d} \varepsilon .
$$

From the Kramers-Kronig relation

$$
\operatorname{Re} \chi_{\mathbf{q}}^{\alpha}(0)=\frac{1}{\pi} \int \frac{\operatorname{Im} \chi_{\mathbf{q}}^{\alpha}(\varepsilon)}{\varepsilon} \mathrm{d} \varepsilon
$$

we obtain the high-temperature form of the fluctuationdissipation theorem:

$$
\left\langle s_{\mathbf{q}}^{\alpha} s_{-\mathbf{q}}^{\alpha}\right\rangle=\frac{T}{2} \chi_{\mathbf{q}}^{\alpha}(0) .
$$

Using formula (1), we write relation (9) as

$$
\frac{1}{N} \chi_{\mathbf{q}}^{\alpha}(0) \frac{1}{2} g^{2} \mu_{\mathrm{B}}^{2}=\frac{M^{2}(q)}{3 T} .
$$

Taking the latter at $\mathbf{q}=0$ and comparing with the CurieWeiss law

$$
\chi_{\mathrm{CW}}^{\alpha}=\frac{m_{\mathrm{eff}}^{2}}{3\left(T-\Theta_{\mathrm{C}}\right)}, \quad T>\Theta_{\mathrm{C}},
$$

we see that

$$
\chi_{\mathrm{CW}}^{\alpha}=\frac{1}{N} \chi_{0}^{\alpha}(0) \frac{1}{2} g^{2} \mu_{\mathrm{B}}^{2}, \quad M(0)=m_{\mathrm{eff}},
$$

for $T \gg \Theta_{\mathrm{C}}$. In the DSFT, calculating the static susceptibility $\chi_{\mathbf{q}}^{\alpha}(0)$ on the right-hand side of (9), we obtain that the spin correlator is given by the Lorentzian function

$$
\left\langle s_{\mathbf{q}}^{\alpha} s_{-\mathbf{q}}^{\alpha}\right\rangle=\frac{N T}{2 u \lambda_{\mathrm{L}}^{\alpha}} \frac{1}{a_{\alpha}^{2}+b_{\alpha}^{2}\left(q / q_{\mathrm{B}}\right)^{2}} .
$$

This high-temperature formula differs from the exact one in the DSFT (8) by the factor with the arctangent.

Fig. 2 shows the square of the effective moment (1) for bcc Fe calculated in the DSFT. We see that the exact formula (8) and high-temperature formula (11) give qualitatively similar results.

Next, we consider the high-temperature approximation of the spatial correlator. At large distances $r>\pi / q_{\mathrm{B}}$, we can extend the integration interval in (4) to infinity. Then the spin correlator reduces to the Ornstein-Zernike form

$$
\left\langle s^{\alpha}(\mathbf{r}) s^{\alpha}(0)\right\rangle=\frac{B}{q_{\mathrm{B}} r} \mathrm{e}^{-r / r_{\mathrm{c}}} .
$$

Here $r_{\mathrm{c}}=\left|b_{\alpha}\right| /\left(\left|a_{\alpha}\right| q_{\mathrm{B}}\right)$ is the correlation radius, which can be considered as a measure of SRO. In the DSFT, it was shown theoretically that $r_{\mathrm{c}}$ increases to infinity as $T$ goes to $T_{\mathrm{C}}$, and $r_{\mathrm{c}}$ decreases to zero inversely with temperature: $r_{\mathrm{c}} \sim 1 / T$, at high temperatures $T \gg T_{\mathrm{C}}[10]$.

\section{Polarized magnetic neutron scattering}

We compare the DSFT calculation results with the results of the polarized magnetic neutron scattering experiment. The magnetic contribution is given by

$$
\left(\frac{\mathrm{d}^{2} \sigma^{\uparrow \downarrow}}{\mathrm{d} \Omega^{\prime} \mathrm{d} E^{\prime}}\right)_{\|}-\left(\frac{\mathrm{d}^{2} \sigma^{\uparrow \downarrow}}{\mathrm{d} \Omega^{\prime} \mathrm{d} E^{\prime}}\right)_{\perp}=\left(\frac{\gamma e^{2}}{m_{\mathrm{e}} c^{2}}\right)^{2} \frac{1}{3} \frac{V}{\Omega_{\mathrm{WS}}} \frac{k^{\prime}}{k} S(\kappa, \omega),
$$

where

$$
S(\kappa, \omega)=\sum_{\alpha} \frac{1}{2 \pi \hbar N} \int\left\langle s_{\kappa}^{\alpha}(t) s_{-\kappa}^{\alpha}\right\rangle \mathrm{e}^{\mathrm{i} \omega t} \mathrm{~d} t
$$

is the scattering function and $\boldsymbol{\kappa}=\mathbf{k}-\mathbf{k}^{\prime}$ is the scattering vector (for details, see [12]). In the experiment [1] the integral scattering over all energies is measured:

$$
\left(\frac{\mathrm{d} \sigma^{\uparrow \downarrow}}{\mathrm{d} \Omega^{\prime}}\right)_{\|}-\left(\frac{\mathrm{d} \sigma^{\uparrow \downarrow}}{\mathrm{d} \Omega^{\prime}}\right)_{\perp}=\left(\frac{\gamma e^{2}}{m_{\mathrm{e}} c^{2}}\right)^{2} \frac{1}{3 g^{2} \mu_{\mathrm{B}}^{2}} \frac{V}{\Omega_{\mathrm{WS}}} \frac{k^{\prime}}{k} M^{2}(\kappa),
$$




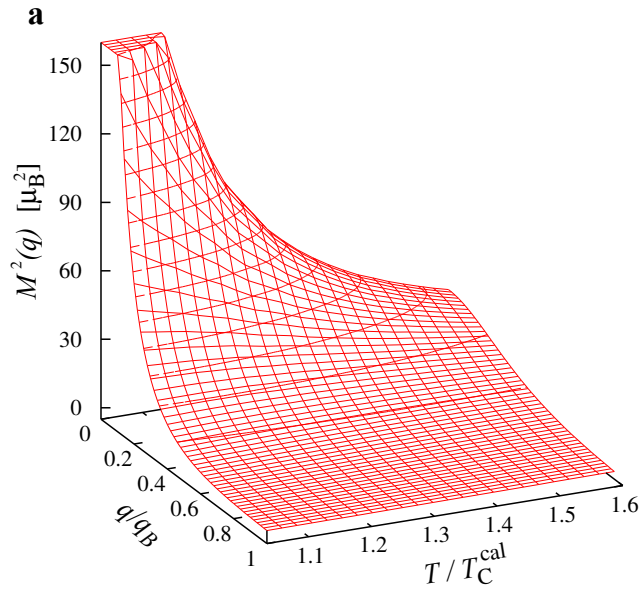

b

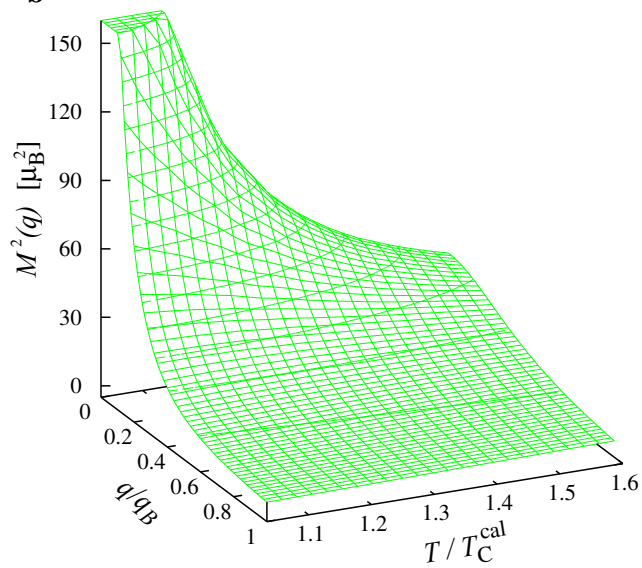

Figure 1. Square of the effective moment for bcc Fe calculated by the exact formula (a) and high-temperature formula (b) in the DSFT.

where $M^{2}(\kappa)$ is the square of the effective moment (1). It is related to the scattering function (12) by the formula

$$
M^{2}(\kappa)=\left(\hbar \int S(\kappa, \omega) \mathrm{d} \omega\right) g^{2} \mu_{\mathrm{B}}^{2} .
$$

Comparing the theoretical results with experimental results, one should keep in mind three circumstances. The first one is the spatial spin distribution, which leads to the magnetic form-factor

$$
F(\mathbf{q}, \mathbf{k}) \approx F(q)=\int|w(\mathbf{r})|^{2} \mathrm{e}^{-\mathrm{i} \mathbf{q r}} \mathrm{d} \mathbf{r},
$$

where $w(\mathbf{r})$ is a Wannier state. For bcc Fe, $\left|F^{2}(q)\right| \approx 0.81$. The second is vibrations of the crystal lattice. This can be taken into account by the Debye-Waller factor $\mathrm{e}^{-2 W}$. Here

$$
2 W(\kappa)=\frac{1}{3} \kappa^{2}\left\langle u^{2}\right\rangle
$$

where $\left\langle u^{2}\right\rangle$ is the mean-square atomic displacement. For bcc Fe, within the Brillouin zone $\mathrm{e}^{-2 W} \approx 1$ (for details, see [14]). The third is the energy cutoff $\varepsilon \leq \varepsilon_{\max }$ in the scattering experiment. To be consistent with the experiment, we integrate over the same energy interval

$$
\left\langle s_{\mathbf{q}}^{\alpha} s_{-\mathbf{q}}^{\alpha}\right\rangle=\frac{1}{2 \pi} \int_{0}^{\varepsilon_{\max }} B(\varepsilon) \operatorname{Im} \chi_{\mathbf{q}}^{\alpha}(\varepsilon) \mathrm{d} \varepsilon
$$

In the experiment [1], the energy cutoff is $\varepsilon_{\max }=50 \mathrm{meV}$.

Comparison of the squared effective moment calculated with the energy cutoff shows good agreement with experiment over a wide range of temperatures above $T_{\mathrm{C}}$ (Fig. 2; for detailed results, see [11]). Results of the exact formula (8) and approximate high-temperature formula (11) can be viewed as a theoretical prediction.

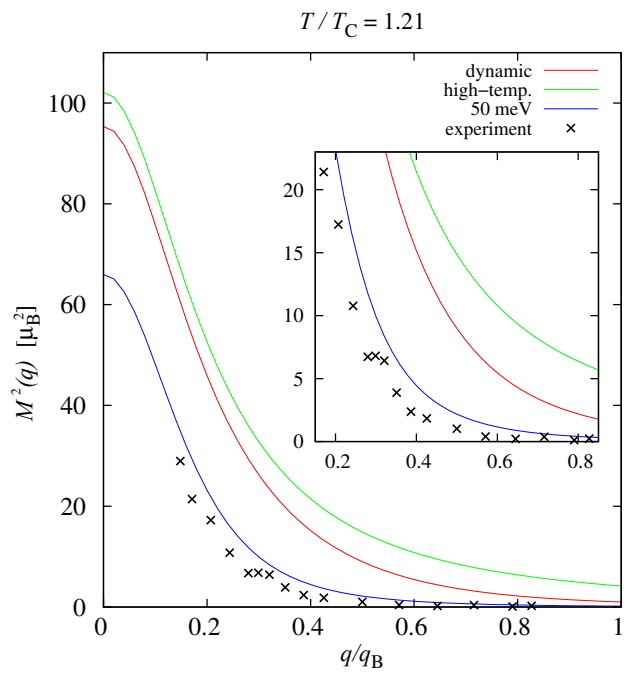

Figure 2. Square of the effective moment (1) for bcc Fe calculated by exact formula (8), approximate high-temperature formula (11) and with the energy cutoff $\varepsilon_{\max }=50 \mathrm{meV}$ compared with the experimental values [1]. The inset shows a region with the experimental points on a larger scale.

The square of the local magnetic moment $m_{\mathrm{L}}^{2}$ is calculated by formula (6), where the spin correlator is given by the exact formula (8), high-temperature formula (11) and formula with the energy cutoff (13). The integrand of (6) is shown in Fig. 3. Results of the calculation for bcc Fe are presented in Table 1. The local moment calculated with the energy cutoff is in reasonable agreement with the experimental one $m_{\mathrm{L}}^{\exp }=1.3 \mu_{\mathrm{B}}$ at $T / T_{\mathrm{C}}^{\exp }=1.2$ [1]. The value $m_{\mathrm{L}}$ calculated with the energy cutoff decreases with increasing temperature (Table 1, last column). This implies that the energy window in the experiment should be wider to include all magnetic scattering, especially at higher temperatures. Calculations by the exact formula are in agreement with the ones using effective Hamiltonians with classical spins, e.g. $m_{\mathrm{L}} \approx 2.2 \mu_{\mathrm{B}}$ at $T / T_{\mathrm{C}}^{\exp }=1.1-1.5$ [15]. Comparing the second and third columns in Table 1, we see that, despite qualitative similarities, the exact and high-temperature formulae give different quantitative results.

In paper [1] the experiment was interpreted based on the spin wave theory. The idea is as follows. The normalized correlation function is given by

$C(r, T)=\left(\int_{0}^{q_{\mathrm{B}}} q^{2} M^{2}(q) \mathrm{d} q\right)^{-1} \int_{0}^{q_{\mathrm{B}}} q^{2} M^{2}(q) \frac{\sin (q r)}{q r} \mathrm{~d} q$. 


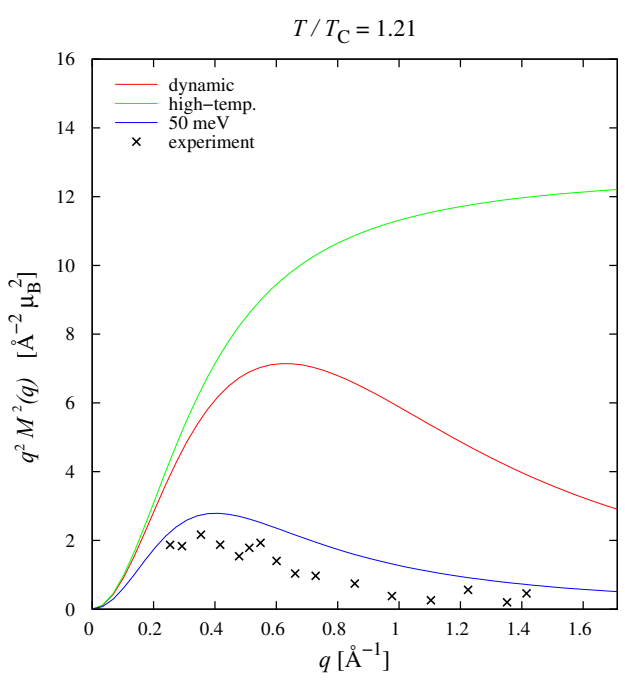

Figure 3. Values of $q^{2} M^{2}(q)$ for bcc Fe (the legend as in Fig. 2).

\begin{tabular}{lccc}
\hline$T / T_{\mathrm{C}}^{\mathrm{cal}}$ & exact, $\mu_{\mathrm{B}}$ & high-temp., $\mu_{\mathrm{B}}$ & $50 \mathrm{meV}, \mu_{\mathrm{B}}$ \\
\hline 1.226 & 2.214 & 3.060 & 1.120 \\
1.322 & 2.218 & 3.080 & 1.116 \\
1.418 & 2.222 & 3.097 & 1.047 \\
1.514 & 2.228 & 3.114 & 0.993 \\
\hline
\end{tabular}

Table 1. Local moment $m_{\mathrm{L}}$ of bcc Fe calculated in the DSFT.

Assuming that $q^{2} M^{2}(q) \propto \delta\left(q-q_{0}\right)$, we obtain a spin wave of the wavelength $\lambda_{0}=2 \pi / q_{0}$ :

$$
C(r, T)=\int_{0}^{q_{\mathrm{B}}} \delta\left(q-q_{0}\right) \frac{\sin (q r)}{q r} \mathrm{~d} q=\frac{\sin \left(q_{0} r\right)}{q_{0} r} .
$$

In bcc Fe $\lambda_{0} \approx 18 \AA$ at $T=1.21 T_{\mathrm{C}}^{\exp }$. The problem with this interpretation is that the peak is too wide: the height and width of the peak are about equal (see Fig. 3).

Instead of approximating the normalized correlation function by a delta-peak, we calculate it in the DSFT (Fig. 4). Since Ornstein-Zernike correlation radius $r_{\mathrm{c}}$ works only at large distances, we measure the SRO domain using the halfwidth $r_{1 / 2}(T)$ of the correlation function $C(r, T)$.

The value of the halfwidth $r_{1 / 2}(T)$ lies within $5 \AA$ in all approximations (Fig. 5). The halfwidth decreases with temperature and comes down to $1-2 \AA$ at $1.6 T / T_{\mathrm{C}}^{\mathrm{cal}}$. From the inset of Fig. 5 we see that the reciprocal halfwidth has an almost linear temperature dependence similarly to the theoretical result for the correlation radius. Moreover, this linear dependence is already observed from about $1.1 T / T_{\mathrm{C}}^{\mathrm{cal}}$.

\section{Conclusions}

In the DSFT, we have calculated the effective moment $M(q)$ as a function of $q$ and $T$ and spin correlator $\langle\mathbf{s}(\mathbf{r}) \mathbf{s}(0)\rangle$ as a function of $r$ and $T$. At high temperatures, $M(q)$ is given by the Lorentzian function, spin correlator has

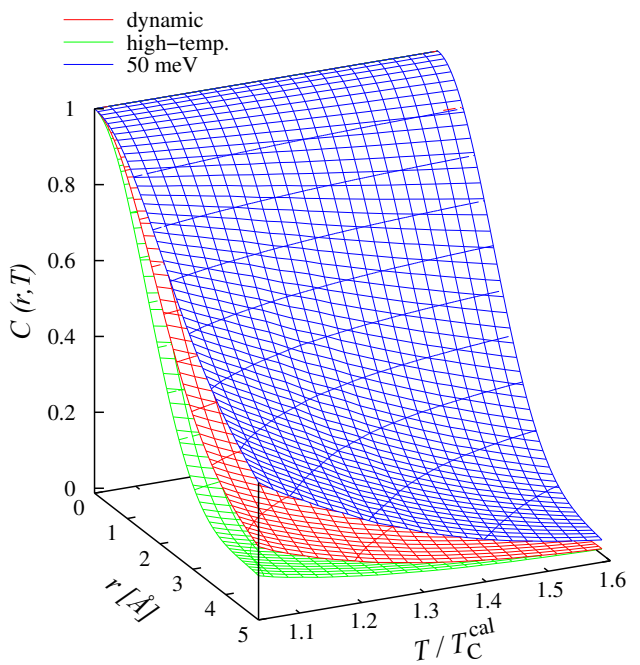

Figure 4. Correlation function $C(r, T)$ of bcc Fe calculated in the DSFT.

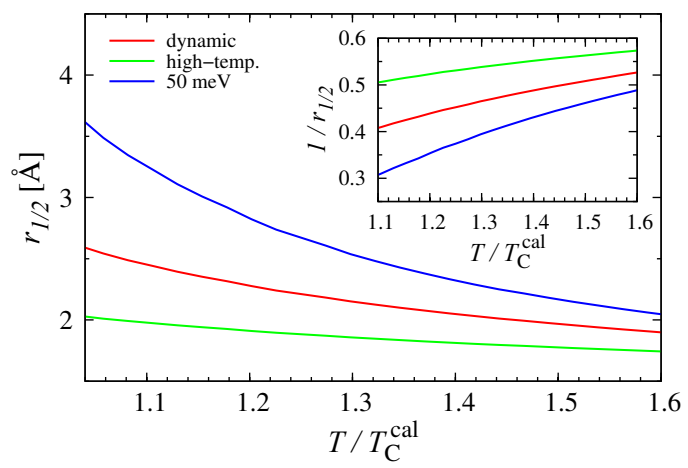

Figure 5. Halfwidth $r_{1 / 2}(T)$ of correlation function $C(r, T)$ for bcc Fe calculated in the DSFT. The inset shows the inverse halfwidth $r_{1 / 2}^{-1}(T)$.

the Ornstein-Zernike form, and $M(0)$ tends to $m_{\mathrm{eff}}$ in the Curie-Weiss law. Calculated $M(q)$ and $m_{\mathrm{L}}$ of bcc Fe above $T_{\mathrm{C}}$ are in good agreement with results of the polarized neutron scattering experiment. Calculated spatial correlator in bcc Fe above $T_{\mathrm{C}}$ gives a small SRO domain (less than $5 \AA$ ). The SRO remains at high temperatures.

\section{Acknowledgments}

The research was carried out within the state assignment of the Federal Agency for Scientific Organizations of Russia (program "Electron" No. 01201463326) and was supported in part by the Program of Ural Branch of Russian Academy of Sciences (Project No. 15-8-2-10).

\section{References}

[1] P. Brown, H. Capellmann, J. Deportes, D. Givord, K. Ziebeck, J. Magn. Magn. Mater. 30, 243 (1982)

[2] G. Shirane, P. Böni, J.P. Wicksted, Phys. Rev. B 33, 1881 (1986) 
[3] T. Moriya, Spin Fluctuations in Itinerant Electron Magnetism (Springer, Berlin, 1985)

[4] A.I. Lichtenstein, M.I. Katsnelson, G. Kotliar, Phys. Rev. Lett. 87, 067205 (2001)

[5] Y. Kakehashi, M.A.R. Patoary, J. Phys. Soc. Jpn. 80, 034706 (2011)

[6] V.I. Grebennikov, Phys. Solid State 40, 79 (1998)

[7] B.I. Reser, V.I. Grebennikov, Phys. Met. Metallogr. 85, 20 (1998)

[8] N.B. Melnikov, B.I. Reser, V.I. Grebennikov, J. Phys.: Condens. Matter 23, 276003 (2011)

[9] V.I. Grebennikov, J. Magn. Magn. Mater. 84, 59 (1990)
[10] N.B. Melnikov, B.I. Reser, J. Magn. Magn. Mater. 397, 347 (2016)

[11] N.B. Melnikov, B.I. Reser, G.V. Paradezhenko, J. Magn. Magn. Mater. 411, 133 (2016)

[12] N.B. Melnikov, G.V. Paradezhenko, B.I. Reser, Theor. Math. Phys. 191, 602 (2017)

[13] N.B. Melnikov, B.I. Reser, Phys. Met. Metallogr. 117, 1328 (2016)

[14] G.V. Paradezhenko, N.B. Melnikov, B.I. Reser, EPJ Web of Conferences (2017), this volume

[15] P.-W. Ma, S.L. Dudarev, Phys. Rev. B 86, 054416 (2012) 